\title{
Bifurcation Analysis and Chaos Control in a Discrete-Time Predator-Prey System of Leslie Type with Simplified Holling Type IV Functional Response
}

\author{
S. M. Sohel Rana ${ }^{1}$ and Umme Kulsum ${ }^{2}$ \\ ${ }^{1}$ Department of Mathematics, University of Dhaka, Dhaka 1000, Bangladesh \\ ${ }^{2}$ Department of Computer Science and Engineering, University of Asia Pacific, Dhaka 1215, Bangladesh
}

Correspondence should be addressed to S. M. Sohel Rana; srana.mthdu@gmail.com

Received 30 March 2017; Accepted 4 June 2017; Published 25 July 2017

Academic Editor: Qamar Din

Copyright (C) 2017 S. M. Sohel Rana and Umme Kulsum. This is an open access article distributed under the Creative Commons Attribution License, which permits unrestricted use, distribution, and reproduction in any medium, provided the original work is properly cited.

\begin{abstract}
The dynamic behavior of a discrete-time predator-prey system of Leslie type with simplified Holling type IV functional response is examined. We algebraically show that the system undergoes a bifurcation (flip or Neimark-Sacker) in the interior of $\mathbb{R}_{+}^{2}$. Numerical simulations are presented not only to validate analytical results but also to show chaotic behaviors which include bifurcations, phase portraits, period 2, 4, 6, 8, 10, and 20 orbits, invariant closed cycle, and attracting chaotic sets. Furthermore, we compute numerically maximum Lyapunov exponents and fractal dimension to justify the chaotic behaviors of the system. Finally, a strategy of feedback control is applied to stabilize chaos existing in the system.
\end{abstract}

\section{Introduction}

In ecology and mathematical biology, the dynamics of predator-prey interaction when the growth of predator depends on the ratio of predator and prey have been extensively investigated by ecologist and mathematician $[1,2]$ and the reference therein. Qualitative analyses of these works found many rich dynamics which include limit cycle, states of stability, codimension 1 subcritical Hopf bifurcation, codimension 2 Bogdanov-Takens bifurcation, and codimension 3 degenerate focus type Bogdanov-Takens bifurcation around positive equilibrium. But lots of exploratory works have suggested that the discretization of predator-prey models is more suitable compared to continuous ones when size of populations is small [3-10]. These researches have mainly focused on Gauss-type predator-prey interaction with monotonic functional responses. They obtained many complex properties in discrete-time models including the possibility of bifurcations (flip and Neimark-Sacker) and chaos phenomenon which had been derived either by using numerical simulations or by using center manifold theory.
In recent times, a few number of articles in literature discussed the dynamics of discrete-time predator-prey systems of Leslie type [11, 12]. For example, a discretetime predator-prey system of Holling and Leslie type with constant-yield prey harvesting was investigated in [11], and a discrete predator-prey model with Holling-Tanner functional response was studied in [12]. In their studies, the authors paid their attention to drive the existence of flip and NeimarkSacker bifurcations by using center manifold theory.

In this paper, we consider the following predator-prey system of Leslie type [1]:

$$
\begin{aligned}
& \dot{x}=r x\left(1-\frac{x}{K}\right)-\frac{m x y}{x^{2}+b}, \\
& \dot{y}=s y\left(1-\frac{y}{h x}\right),
\end{aligned}
$$

where $x$ and $y$ stand for densities of prey and predator, respectively; $r, K, m, b, s$, and $h$ are all positive constants, and $m x y /\left(x^{2}+b\right)$ denotes the functional response of simplified Holling type IV. In [1], it is shown that model (1) has a 
degenerate Bogdanov-Takens bifurcation of codimension 3 at positive equilibrium. For simplicity, we scale the variables and parameters as

$$
\begin{aligned}
\frac{x}{K} & \longrightarrow x, \\
\frac{m y}{r K^{2}} & \longrightarrow y, \\
r t & \longrightarrow t, \\
a & =\frac{b}{K^{2}}, \\
\alpha & =\frac{s}{r}, \\
\beta & =\frac{s K}{h m} .
\end{aligned}
$$

Then we write system (1) in the form

$$
\begin{aligned}
& \dot{x}=x(1-x)-\frac{x y}{x^{2}+a}, \\
& \dot{y}=y\left(\alpha-\frac{\beta y}{x}\right) .
\end{aligned}
$$

Forward Euler scheme is applied to system (3) to get the following discrete system:

$$
\left(\begin{array}{l}
x \\
y
\end{array}\right) \longmapsto\left(\begin{array}{c}
x+\delta x\left[(1-x)-\frac{y}{x^{2}+a}\right] \\
y+\delta y\left[\alpha-\frac{\beta y}{x}\right]
\end{array}\right),
$$

where $\delta$ is the step size. The objective is to study systematically the existence condition of a bifurcation (flip or NS bifurcation) in the interior of $\mathbb{R}_{+}^{2}$ using bifurcation theory and center manifold theory [13-16].

This paper is organized as follows. Section 2 deals with the existence condition for fixed points of system (4) and their stability criterion. In Section 3, we prove that under certain parametric condition system (4) admits a bifurcation. In Section 4, we implement numerical simulations of the system for one or more control parameters which include diagrams of bifurcation, phase portraits, maximum Lyapunov exponents, and fractal dimensions. In Section 5, we use the method of feedback control to stabilize chaos at unstable trajectories. Finally we carry out a short discussion in Section 6.

\section{Fixed Points: Existence and Their Stability}

The fixed points of system (4) are solution of

$$
\begin{aligned}
x+\delta x\left[(1-x)-\frac{y}{x^{2}+a}\right] & =x, \\
y+\delta y\left[\alpha-\frac{\beta y}{x}\right] & =y .
\end{aligned}
$$

A simple algebraic computation shows that system (4) has a predator free fixed point $E_{1}(1,0)$ for all parameter values.
Next, it is of great interest to find the positive fixed point of system (4). Suppose that $E_{2}\left(x^{*}, y^{*}\right)$ is a positive fixed point of system (4). Then, $x^{*}$ and $y^{*}$ are solutions of

$$
\begin{aligned}
1-x^{*} & =\frac{y^{*}}{x^{* 2}+a}, \\
\alpha x^{*} & =\beta y^{*} .
\end{aligned}
$$

From (6), we can see that $x^{*} \in(0,1)$ is the root of the following cubic equation:

$$
p_{0} w^{3}+3 p_{1} w^{2}+3 p_{2} w+p_{3}=0, \quad\left(p_{0} \neq 0\right),
$$

with coefficients

$$
\begin{aligned}
p_{0} & =\frac{1}{a}, \\
3 p_{1} & =-\frac{1}{a}, \\
3 p_{2} & =1+\frac{\alpha}{a \beta}, \\
p_{3} & =-1 .
\end{aligned}
$$

Now, the transformation $z=p_{0} w+p_{1}$ converts (7) to $z^{3}+$ $3 H z+G=0$, where $G=p_{0}{ }^{2} p_{3}-3 p_{0} p_{1} p_{2}+2 p_{1}{ }^{3}, H=$ $p_{0} p_{2}-p_{1}{ }^{2}$. Using Cardano's method, we get following result.

Lemma 1. If $G^{2}+4 H^{3}>0$, then a unique positive fixed point $E_{2}\left(x^{*}, y^{*}\right)$ of system (4) exists, where

$$
\begin{aligned}
& x^{*}=\frac{1}{p_{0}}\left(q-\frac{H}{q}-p_{1}\right), \\
& y^{*}=\frac{\alpha x^{*}}{\beta},
\end{aligned}
$$

and $q$ denotes one of the three values of $[(-G+$ $\left.\left.\sqrt{G^{2}+4 H^{3}}\right) / 2\right]^{1 / 3}$.

Next, we investigate stability of system (4) at fixed points. Note that the magnitude of eigenvalues of Jacobian matrix evaluated at fixed point $E(x, y)$ determines the local stability of that fixed point. The Jacobian matrix of system (4) evaluated at fixed point $E(x, y)$ is given by

$$
J(x, y)=\left(\begin{array}{ll}
a_{11} & a_{12} \\
a_{21} & a_{22}
\end{array}\right),
$$

where

$$
\begin{aligned}
& a_{11}=1+\delta-2 \delta x+\frac{\delta y\left(x^{2}-a\right)}{\left(x^{2}+a\right)^{2}}, \\
& a_{12}=-\frac{\delta x}{x^{2}+a}, \\
& a_{21}=\frac{\beta \delta y^{2}}{x^{2}}, \\
& a_{22}=1+\alpha \delta-\frac{2 \beta \delta y}{x} .
\end{aligned}
$$


The characteristic equation of matrix $J$ is

$$
\lambda^{2}+p(x, y) \lambda+q(x, y)=0
$$

where $p(x, y)=-\operatorname{tr} J=-\left(a_{11}+a_{22}\right)$ and $\operatorname{det} J=a_{11} a_{22}-$ $a_{12} a_{21}$.

Using Jury's criterion [17], we state the following stability conditions of fixed points.

Proposition 2. The predator free fixed point $E_{1}(1,0)$ is a saddle if $0<\delta<2$, source if $\delta>2$, and nonhyperbolic if $\delta=2$.

It is obvious that when $\delta=2$, the two eigenvalues of $J\left(E_{1}\right)$ are $\lambda_{1}=1-\delta$ and $\lambda_{2}=1+\alpha \delta>1$. Therefore, a flip bifurcation can occur if parameters change in small vicinity of $\mathrm{FB}_{E_{1}}$ :

$$
\mathrm{FB}_{E_{1}}=\{(a, \alpha, \beta, \delta) \in(0,+\infty): \delta=2\} .
$$

At $E_{2}\left(x^{*}, y^{*}\right)$, we write (12) as

$$
F(\lambda):=\lambda^{2}-(2+\Delta \delta) \lambda+\left(1+\Delta \delta+\Omega \delta^{2}\right)=0,
$$

where

$$
\begin{aligned}
\Delta= & 1-2 x^{*}+\frac{y^{*}\left(x^{* 2}-a\right)}{\left(x^{* 2}+a\right)^{2}}+\alpha-\frac{2 \beta y^{*}}{x^{*}} \\
\Omega= & \left(1-2 x^{*}+\frac{y^{*}\left(x^{* 2}-a\right)}{\left(x^{* 2}+a\right)^{2}}\right) \alpha+\frac{2 \beta y^{*}\left(2 x^{*}-1\right)}{x^{*}} \\
& -\frac{\beta y^{* 2}\left(x^{* 2}-3 a\right)}{x^{*}\left(x^{* 2}+a\right)^{2}} .
\end{aligned}
$$

Then $F(1)=\Omega \delta^{2}>0$ and $F(-1)=4+2 \Delta \delta+\Omega \delta^{2}$.

We state the following Proposition about stability criterion of $E_{2}$.

Proposition 3. Suppose that fixed point $E_{2}\left(x^{*}, y^{*}\right)$ of system (4) exists. Then it is

(i) sink if one of the following conditions holds:

(i.1) $\Delta^{2}-4 \Omega \geq 0$ and $\delta<\left(-\Delta-\sqrt{\Delta^{2}-4 \Omega}\right) / \Omega$;

(i.2) $\Delta^{2}-4 \Omega<0$ and $\delta<-\Delta / \Omega$;

(ii) source if one of the following conditions holds:

(ii.1) $\Delta^{2}-4 \Omega \geq 0$ and $\delta>\left(-\Delta+\sqrt{\Delta^{2}-4 \Omega}\right) / \Omega$;

(ii.2) $\Delta^{2}-4 \Omega<0$ and $\delta>-\Delta / \Omega$;

(iii) nonhyperbolic if one of the following conditions holds:

(iii.1) $\Delta^{2}-4 \Omega \geq 0$ and $\delta=\left(-\Delta \pm \sqrt{\Delta^{2}-4 \Omega}\right) / \Omega$;

(iii.2) $\Delta^{2}-4 \Omega<0$ and $\delta=-\Delta / \Omega$;

(iv) saddle if otherwise.
From Proposition 3, we see that two eigenvalues of $J\left(E_{2}\right)$ are $\lambda_{1}=-1$ and $\lambda_{2} \neq-1,1$ if condition (iii.1) holds. We rewrite term (iii.1) as follows:

$$
\begin{aligned}
\mathrm{FB}_{E_{2}}^{1} & =\{(a, \alpha, \beta, \delta) \in(0,+\infty): \delta \\
& \left.=\frac{-\Delta-\sqrt{\Delta^{2}-4 \Omega}}{\Omega}, \Delta^{2}-4 \Omega \geq 0\right\},
\end{aligned}
$$

or

$$
\begin{aligned}
\mathrm{FB}_{E_{2}}^{2} & =\{(a, \alpha, \beta, \delta) \in(0,+\infty): \delta \\
& \left.=\frac{-\Delta+\sqrt{\Delta^{2}-4 \Omega}}{\Omega}, \Delta^{2}-4 \Omega \geq 0\right\} .
\end{aligned}
$$

Therefore, a flip bifurcation can appear at $E_{2}$ if parameters vary around the set $\mathrm{FB}_{E_{2}}^{1}$ or $\mathrm{FB}_{E_{2}}^{2}$.

Also we rewrite term (iii.2) as follows:

$$
\begin{aligned}
& \mathrm{NSB}_{E_{2}} \\
& \quad=\left\{(a, \alpha, \beta, \delta) \in(0,+\infty): \delta=-\frac{\Delta}{\Omega}, \Delta^{2}-4 \Omega<0\right\},
\end{aligned}
$$

and if the parameters change in small vicinity of $\mathrm{NSB}_{E_{2}}$, two eigenvalues $\lambda_{1,2}$ of $J\left(E_{2}\right)$ are complex having magnitude one and then NS bifurcation can emerge from fixed point $E_{2}$.

\section{Bifurcation Analysis}

In this section, we will give attention to recapitulate bifurcations (flip and Neimark-Sacker) of system (4) around $E_{2}$ by using the theory of bifurcation [13-16]. We set $\delta$ as a bifurcation parameter.

3.1. Flip Bifurcation. Consider system (4) at the fixed point $E_{2}\left(x^{*}, y^{*}\right)$ with arbitrary parameter $(a, \alpha, \beta, \delta) \in \mathrm{FB}_{E_{2}}^{1}$. One can consider the case of $\mathrm{FB}_{E_{2}}^{2}$ in a similar fashion. Since the parameters lie in $\mathrm{FB}_{E_{2}}^{1}$, let $\delta=\delta_{F}=\left(-\Delta-\sqrt{\Delta^{2}-4 \Omega}\right) / \Omega$, and then the eigenvalues of positive fixed point $\left(x^{*}, y^{*}\right)$ are

$$
\begin{aligned}
& \lambda_{1}\left(\delta_{F}\right)=-1, \\
& \lambda_{2}\left(\delta_{F}\right)=3+\Delta \delta_{F} .
\end{aligned}
$$

The condition $\left|\lambda_{2}\left(\delta_{F}\right)\right| \neq 1$ leads to

$$
\Delta \delta_{F} \neq-2,-4 \text {. }
$$

Using the transformation $\tilde{x}=x-x^{*}, \tilde{y}=y-y^{*}$ and writing $A(\delta)=J\left(x^{*}, y^{*}\right)$, we shift the fixed point $\left(x^{*}, y^{*}\right)$ of system (4) to the origin. After Taylor expansion, system (4) reduces to

$$
\left(\begin{array}{l}
\tilde{x} \\
\tilde{y}
\end{array}\right) \longrightarrow A(\delta)\left(\begin{array}{l}
\tilde{x} \\
\tilde{y}
\end{array}\right)+\left(\begin{array}{l}
F_{1}(\tilde{x}, \tilde{y}, \delta) \\
F_{2}(\tilde{x}, \tilde{y}, \delta)
\end{array}\right),
$$


where $X=(\tilde{x}, \tilde{y})^{T}$ and

$$
\begin{aligned}
& F_{1}(\tilde{x}, \tilde{y}, \delta)=\frac{1}{6}\left[\frac{6 \delta y^{*}\left(a^{2}-6 a x^{* 2}+x^{* 4}\right)}{\left(x^{* 2}+a\right)^{4}} \tilde{x}^{3}\right. \\
& \left.+\frac{3 \delta\left(6 a x^{*}-2 x^{* 3}\right)}{\left(x^{* 2}+a\right)^{3}} \tilde{x}^{2} \tilde{y}\right] \\
& +\frac{1}{2}\left[\left(-2 \delta+\frac{\delta y^{*}\left(6 a x^{*}-2 x^{* 3}\right)}{\left(x^{* 2}+a\right)^{3}}\right) \tilde{x}^{2}\right. \\
& \left.+\frac{2 \delta\left(x^{* 2}-a\right)}{\left(x^{* 2}+a\right)^{2}} \tilde{x} \tilde{y}\right]+O\left(\|X\|^{4}\right), \\
& F_{2}(\tilde{x}, \tilde{y}, \delta)=\frac{\beta \delta}{x^{* 4}} \tilde{x}\left(x^{*} \tilde{y}-y^{*} \tilde{x}\right)^{2}-\frac{\beta \delta}{x^{* 3}} \tilde{x}\left(x^{*} \tilde{y}\right. \\
& \left.-y^{*} \tilde{x}\right)^{2}+O\left(\|X\|^{4}\right) .
\end{aligned}
$$

It follows that

$$
\begin{aligned}
& B_{1}(x, y)=\left.\sum_{j, k=1}^{2} \frac{\delta^{2} F_{1}(\xi, \delta)}{\delta \xi_{j} \delta \xi_{k}}\right|_{\xi=0} x_{j} y_{k} \\
& =\frac{\delta\left(x^{* 2}-a\right)}{\left(x^{* 2}+a\right)^{2}}\left(x_{1} y_{2}+x_{2} y_{1}\right) \\
& +\left(-2 \delta+\frac{\delta y^{*}\left(6 a x^{*}-2 x^{* 3}\right)}{\left(x^{* 2}+a\right)^{3}}\right) x_{1} y_{1}, \\
& B_{2}(x, y)=\left.\sum_{j, k=1}^{2} \frac{\delta^{2} F_{2}(\xi, \delta)}{\delta \xi_{j} \delta \xi_{k}}\right|_{\xi=0} x_{j} y_{k} \\
& =-\frac{2 \beta \delta}{x^{*}} x_{2} y_{2}+\frac{2 \beta \delta y^{*}}{x^{* 2}}\left(x_{1} y_{2}+x_{2} y_{1}\right) \\
& \quad-\frac{2 \beta \delta y^{* 2}}{x^{* 3}} x_{1} y_{1}, \\
& C_{1}(x, y, u)=\left.\sum_{j, k, l=1}^{2} \frac{\delta^{2} F_{1}(\xi, \delta)}{\delta \xi_{j} \delta \xi_{k} \delta \xi_{l}}\right|_{\xi=0} x_{j} y_{k} u_{l} \\
& \quad+\frac{6 \delta y^{*}\left(a^{2}-6 a x^{* 2}+x^{* 4}\right)}{\left(x^{* 2}+a\right)^{4}} x_{1} y_{1} u_{1}, \\
& =\frac{\left(6 a x^{*}-2 x^{* 3}\right)}{\left(x^{* 2}+a\right)^{3}}\left(x_{1} y_{2} u_{1}+x_{2} y_{1} u_{1}+x_{1} y_{1} u_{2}\right)
\end{aligned}
$$

$$
\begin{gathered}
C_{2}(x, y, u)=\left.\sum_{j, k, l=1}^{2} \frac{\delta^{2} F_{2}(\xi, \delta)}{\delta \xi_{j} \delta \xi_{k} \delta \xi_{l}}\right|_{\xi=0} x_{j} y_{k} u_{l} \\
=\frac{2 \beta \delta}{x^{* 2}}\left(x_{1} y_{2} u_{2}+x_{2} y_{1} u_{2}+x_{2} y_{2} u_{1}\right) \\
\quad-\frac{4 \beta \delta y^{*}}{x^{* 3}}\left(x_{1} y_{1} u_{2}+x_{1} y_{2} u_{1}+x_{2} y_{1} u_{1}\right) \\
+\frac{6 \beta \delta y^{* 2}}{x^{* 4}} x_{1} y_{1} u_{1},
\end{gathered}
$$

and $\delta=\delta_{F}$.

Therefore, we obtain the following symmetric multilinear vector functions of $x, y, u \in \mathbb{R}^{2}$ :

$$
\begin{gathered}
B(x, y)=\left(\begin{array}{l}
B_{1}(x, y) \\
B_{2}(x, y)
\end{array}\right), \\
C(x, y, u)=\left(\begin{array}{l}
C_{1}(x, y, u) \\
C_{2}(x, y, u)
\end{array}\right) .
\end{gathered}
$$

Let $p, q \in \mathbb{R}^{2}$ be two eigenvectors of $A$ for eigenvalue $\lambda_{1}\left(\delta_{F}\right)=-1$ such that $A\left(\delta_{F}\right) q=-q$ and $A^{T}\left(\delta_{F}\right) p=-p$. Then by direct calculation we get

$$
\begin{aligned}
& q \sim\left(2+\alpha \delta_{F}-\frac{2 \beta \delta_{F} y^{*}}{x^{*}},-\frac{\beta \delta_{F} y^{* 2}}{x^{* 2}}\right)^{T}, \\
& p \sim\left(2+\alpha \delta_{F}-\frac{2 \beta \delta_{F} y^{*}}{x^{*}}, \frac{\delta_{F} x^{*}}{x^{* 2}+a}\right)^{T} .
\end{aligned}
$$

We set $p=\gamma_{1}\left(2+\alpha \delta_{F}-2 \beta \delta_{F} y^{*} / x^{*}, \delta_{F} x^{*} /\left(x^{* 2}+a\right)\right)^{T}$, where $\gamma_{1}$

$$
=\frac{1}{\left(2+\alpha \delta_{F}-2 \beta \delta_{F} y^{*} / x^{*}\right)^{2}-\beta \delta_{F}^{2} y^{* 2} / x^{*}\left(x^{* 2}+a\right)} .
$$

Then by the standard scalar product in $\mathbb{R}^{2}$ defined by $\langle p, q\rangle=p_{1} q_{1}+p_{2} q_{2}$, we show that $\langle p, q\rangle=1$. The direction of the flip bifurcation is obtained by sign $c\left(\delta_{F}\right)$, the coefficient of critical normal form [13], and is given by

$$
\begin{aligned}
c\left(\delta_{F}\right)= & \frac{1}{6}\langle p, C(q, q, q)\rangle \\
& -\frac{1}{2}\left\langle p, B\left(q,(A-I)^{-1} B(q, q)\right)\right\rangle .
\end{aligned}
$$

We state the following result on flip bifurcation according to the above analysis.

Theorem 4. If (20) holds, $c\left(\delta_{F}\right) \neq 0$, and the parameter $\delta$ changes its value around $\delta_{F}$, then system (4) undergoes a flip bifurcation at positive fixed point $E_{2}\left(x^{*}, y^{*}\right)$. Moreover, the period 2 orbits that bifurcate from $E_{2}\left(x^{*}, y^{*}\right)$ are stable (resp., unstable) if $c\left(\delta_{F}\right)>0\left(\right.$ resp., $\left.c\left(\delta_{F}\right)<0\right)$. 
3.2. Neimark-Sacker Bifurcation. We consider system (4) at fixed point $E_{2}\left(x^{*}, y^{*}\right)$ with arbitrary $(a, \alpha, \beta, \delta) \in \mathrm{NSB}_{E_{2}}$. From (12), the eigenvalues are given by

$$
\lambda, \bar{\lambda}=\frac{-p(\delta) \pm \sqrt{p(\delta)^{2}-4 q(\delta)}}{2} .
$$

Since the parameters belong to $\mathrm{NSB}_{E_{2}}$, the eigenvalues will be complex and

$$
\lambda, \bar{\lambda}=1+\frac{\Delta \delta}{2} \pm \frac{i \delta}{2} \sqrt{4 \Omega-\Delta^{2}} .
$$

Let

$$
\delta=\delta_{\mathrm{NS}}=-\frac{\Delta}{\Omega}
$$

Therefore, we have

$$
\begin{aligned}
|\lambda| & =\sqrt{q(\delta),} \\
q\left(\delta_{\mathrm{NS}}\right) & =1, \\
\left.\frac{d|\lambda(\delta)|}{d \delta}\right|_{\delta=\delta_{\mathrm{NS}}} & =-\frac{\Delta}{2} \neq 0 .
\end{aligned}
$$

Moreover, if $p\left(\delta_{\mathrm{NS}}\right) \neq 0,1$, then

$$
\frac{\Delta^{2}}{\Omega} \neq 2,3
$$

which obviously satisfies

$$
\lambda^{k}\left(\delta_{\mathrm{NS}}\right) \neq 1 \quad \text { for } k=1,2,3,4 .
$$

Suppose that $q, p \in \mathbb{C}^{2}$ are two eigenvectors of $A\left(\delta_{\mathrm{NS}}\right)$ and $A^{T}\left(\delta_{\mathrm{NS}}\right)$ for eigenvalues $\lambda\left(\delta_{\mathrm{NS}}\right)$ and $\bar{\lambda}\left(\delta_{\mathrm{NS}}\right)$ such that

$$
\begin{array}{r}
A\left(\delta_{\mathrm{NS}}\right) q=\lambda\left(\delta_{\mathrm{NS}}\right) q, \\
A\left(\delta_{\mathrm{NS}}\right) \bar{q}=\bar{\lambda}\left(\delta_{\mathrm{NS}}\right) \bar{q}, \\
A^{T}\left(\delta_{\mathrm{NS}}\right) p=\bar{\lambda}\left(\delta_{\mathrm{NS}}\right) p, \\
A^{T}\left(\delta_{\mathrm{NS}}\right) \bar{p}=\lambda\left(\delta_{\mathrm{NS}}\right) \bar{p} .
\end{array}
$$

Then by direct computation we obtain

$$
\begin{aligned}
& q \sim\left(1+\alpha \delta_{\mathrm{NS}}-\frac{2 \beta \delta y^{*}}{x^{*}}-\lambda,-\frac{\beta \delta y^{* 2}}{x^{* 2}}\right)^{T}, \\
& p \sim\left(1+\alpha \delta_{\mathrm{NS}}-\frac{2 \beta \delta y^{*}}{x^{*}}-\bar{\lambda}, \frac{\delta x^{*}}{x^{* 2}+a}\right)^{T} .
\end{aligned}
$$

We set $p=\gamma_{2}\left(1+\alpha \delta_{\mathrm{NS}}-2 \beta \delta y^{*} / x^{*}-\bar{\lambda}, \delta x^{*} /\left(x^{* 2}+a\right)\right)^{T}$, where

$$
\begin{aligned}
& \gamma_{2} \\
& =\frac{1}{\left(1+\alpha \delta_{\mathrm{NS}}-2 \beta \delta y^{*} / x^{*}-\bar{\lambda}\right)^{2}-\beta \delta_{\mathrm{NS}}^{2} y^{* 2} / x^{*}\left(x^{* 2}+a\right)} .
\end{aligned}
$$

Then it is clear that $\langle p, q\rangle=1$ where $\langle p, q\rangle=\overline{p_{1}} q_{2}+\overline{p_{2}} q_{1}$ for $p, q \in \mathbb{C}^{2}$. Now, we decompose vector $X \in \mathbb{R}^{2}$ as $X=z q+$ $\bar{z} \bar{q}$, for $\delta$ close to $\delta_{\mathrm{NS}}$ and $z \in \mathbb{C}$. Obviously, $z=\langle p, X\rangle$. Thus, we obtain the following transformed form of system (21) for $|\delta|$ near $\delta_{\mathrm{NS}}$ :

$$
z \longmapsto \lambda(\delta) z+g(z, \bar{z}, \delta)
$$

where $\lambda(\delta)=(1+\varphi(\delta)) e^{i \theta(\delta)}$ with $\varphi\left(\delta_{\mathrm{NS}}\right)=0$ and $g(z, \bar{z}, \delta)$ is a smooth complex-valued function. After Taylor expression of $g$ with respect to $(z, \bar{z})$, we obtain

$$
g(z, \bar{z}, \delta)=\sum_{k+l \geq 2} \frac{1}{k ! l !} g_{k l}(\delta) z^{k} \bar{z}^{l}
$$

with $g_{k l} \in \mathbb{C}, k, l=0,1, \ldots$.

According to multilinear symmetric vector functions, the coefficients $g_{k l}$ are

$$
\begin{aligned}
& g_{20}\left(\delta_{\mathrm{NS}}\right)=\langle p, B(q, q)\rangle, \\
& g_{11}\left(\delta_{\mathrm{NS}}\right)=\langle p, B(q, \bar{q})\rangle, \\
& g_{02}\left(\delta_{\mathrm{NS}}\right)=\langle p, B(\bar{q}, \bar{q})\rangle, \\
& g_{21}\left(\delta_{\mathrm{NS}}\right)=\langle p, C(q, q, \bar{q})\rangle .
\end{aligned}
$$

The invariant closed curve appear in the direction which is determined by the coefficient $a\left(\delta_{\mathrm{NS}}\right)$ and calculated via

$$
\begin{aligned}
a\left(\delta_{\mathrm{NS}}\right)= & \operatorname{Re}\left(\frac{e^{-i \theta\left(\delta_{\mathrm{NS}}\right)} g_{21}}{2}\right) \\
& -\operatorname{Re}\left(\frac{\left(1-2 e^{i \theta\left(\delta_{\mathrm{NS}}\right)}\right) e^{-2 i \theta\left(\delta_{\mathrm{NS}}\right)}}{2\left(1-e^{i \theta\left(\delta_{\mathrm{NS}}\right)}\right)} g_{20} g_{11}\right) \\
& -\frac{1}{2}\left|g_{11}\right|^{2}-\frac{1}{4}\left|g_{02}\right|^{2},
\end{aligned}
$$

where $e^{i \theta\left(\delta_{\mathrm{NS}}\right)}=\lambda\left(\delta_{\mathrm{NS}}\right)$.

It is clear that conditions (31) and (33) known as transversal and nondegenerate for system (4) hold well. We obtain the following result.

Theorem 5. If (32) holds, $a\left(\delta_{N S}\right) \neq 0$, and the parameter $\delta$ changes its value in small vicinity of $\mathrm{NSB}_{\mathrm{E}_{2}}$, then system (4) passes through a Neimark-Sacker bifurcation at positive fixed point $E_{2}$. Moreover, if $a\left(\delta_{N S}\right)<0$ (resp., $\left.>0\right)$, then there exists a unique attracting (resp., repelling) invariant closed curve which bifurcates from $E_{2}$.

\section{Numerical Simulations}

Here, diagrams for bifurcation, phase portraits, maximum Lyapunov exponents, and fractal dimension of system (4) will be drawn to validate our theoretical results using numerical simulation. We consider parameter values in the following cases for bifurcation analysis. 


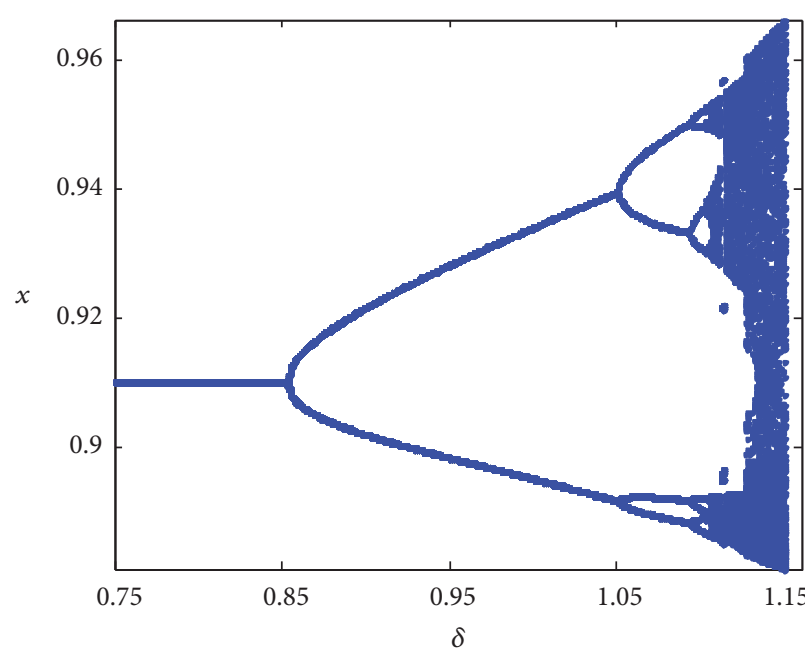

(a)

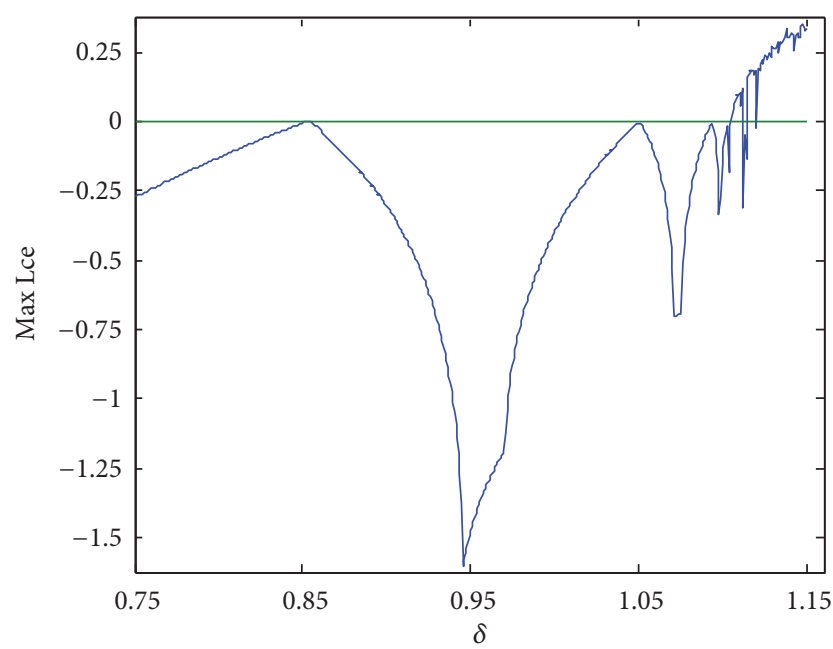

(c)

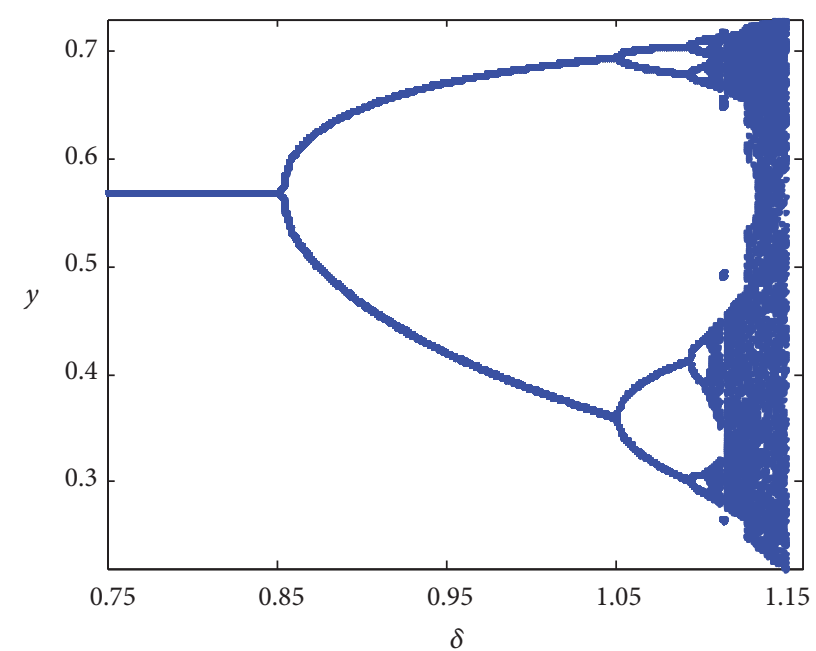

(b)

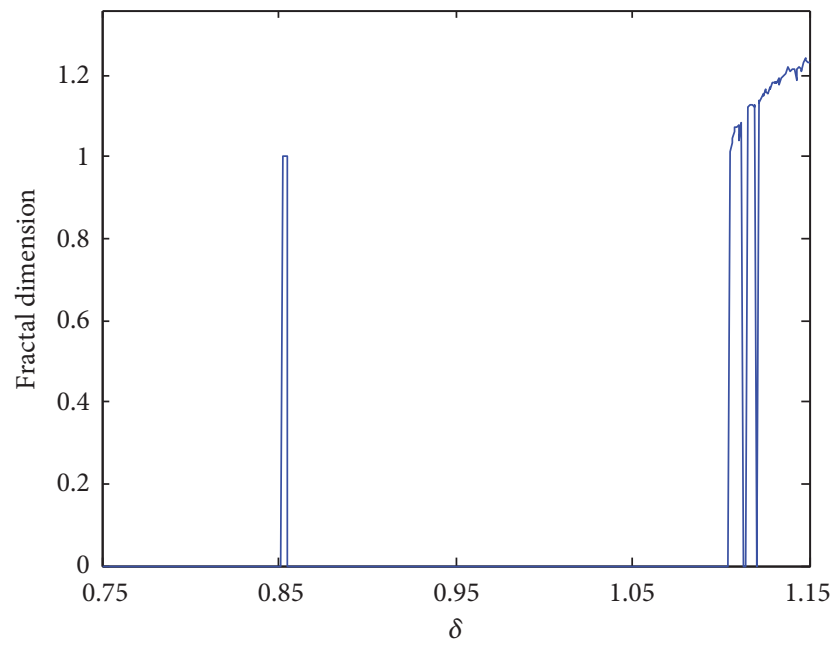

(d)

FIgURE 1: Flip bifurcation and Lyapunov exponent of system (4). (a) Bifurcation for prey, (b) bifurcation for predator, (c) maximum Lyapunov exponents related to $(\mathrm{a}-\mathrm{b})$, and (d) fractal dimension corresponding to (a). Initial value $\left(x_{0}, y_{0}\right)=(0.9,0.56)$.

Case 1. Vary $\delta$ in range $0.75 \leq \delta \leq 1.17$, and fix $a=5.5, \alpha=$ $2.5, \beta=4.0$.

Case 2. Vary $\delta$ in range $1.9 \leq \delta \leq 2.15$, and fix $a=2.5, \alpha=$ $0.75, \beta=0.5$.

Case 3. Vary $\delta$ in range $1.9 \leq \delta \leq 2.15, \beta$ in range $0.5 \leq \beta \leq$ 0.8 , and fix $a=2.5, \alpha=0.75$.

For Case 1, taking parameters $a=5.5, \alpha=2.5$, and $\beta=4.0$ and $\delta$ covering $[0.75,1.17]$, we find that, at fixed point $E_{2}(0.910115,0.568822)$, a flip bifurcation occurs at $\delta=\delta_{F} \sim$ 0.852505 with multipliers $\lambda_{1}=-1, \lambda_{2}=0.112921, a\left(\delta_{F}\right)=$ 22.3878 , and $(a, \alpha, \beta, \delta) \in \mathrm{FB}_{E_{2}}^{1}$. This verifies Theorem 4 .

According to bifurcation diagrams shown in Figures 1(a) and $1(\mathrm{~b})$, we see that stability of fixed point $E_{2}$ happens for $\delta<0.852505$ and loses its stability at $\delta=0.852505$ and period doubling phenomena lead to chaos for $\delta>0.852505$. The maximum Lyapunov exponents and fractal dimension related to Figures 1(a) and 1(b) are computed and shown in Figures 1(c) and 1(d). We observe that the period 2, 4, and 8 orbits occur for $\delta \in[0.75,1.104]$, chaotic set occurs for $\delta \in[1.104,1.15]$, and the period 6 orbit occurs at $\delta=1.112$ within the window of chaotic region $\delta \in[1.104,1.15]$. The status of stable, periodic, or chaotic dynamics is compatible with sign in Figures 1(c) and 1(d).

For Case 2, taking parameters $a=2.5, \alpha=0.75$, and $\beta=0.5$ and $\delta$ covering $[1.9,2.15]$, we observe that a Neimark-Sacker (NS) bifurcation appears at fixed point $(0.662032,0.993048)$ for $\delta=\delta_{\mathrm{NS}} \sim 1.94431$. Also, we have $\lambda, \bar{\lambda}=-0.274696 \pm 0.961531 i, g_{20}=3.36329-2.22294 i$, $g_{11}=3.85086-1.04183 i, g_{02}=-1.24765+5.18251 i, g_{21}=$ $-4.31749+7.22596 i, a\left(\delta_{\mathrm{NS}}\right)=-11.9921$, and $(a, \alpha, \beta, \delta) \epsilon$ $\mathrm{NSB}_{E_{2}}$. This verifies Theorem 5 .

The bifurcation diagrams shown in Figures 2(a) and 2(b) demonstrate that stability of $E_{2}$ happens for $\delta<1.94431$ and loses its stability at $\delta=1.94431$ and an attracting invariant curve appears if $\delta>1.94431$. We dispose the maximum 


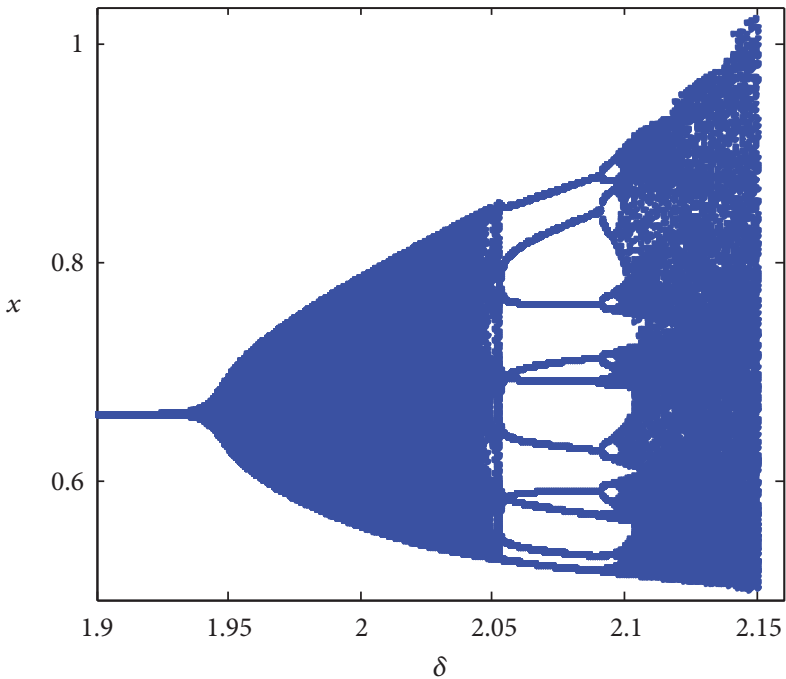

(a)

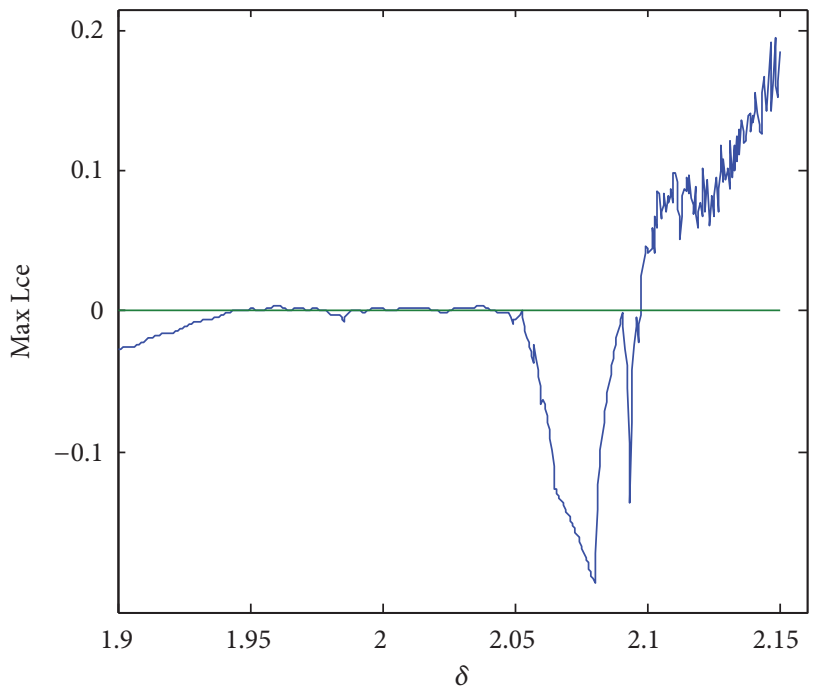

(c)

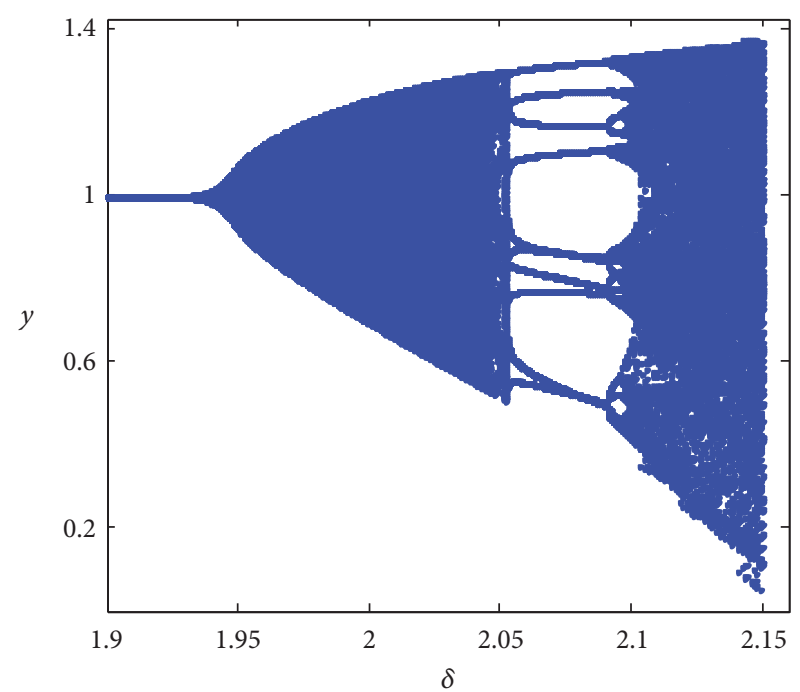

(b)

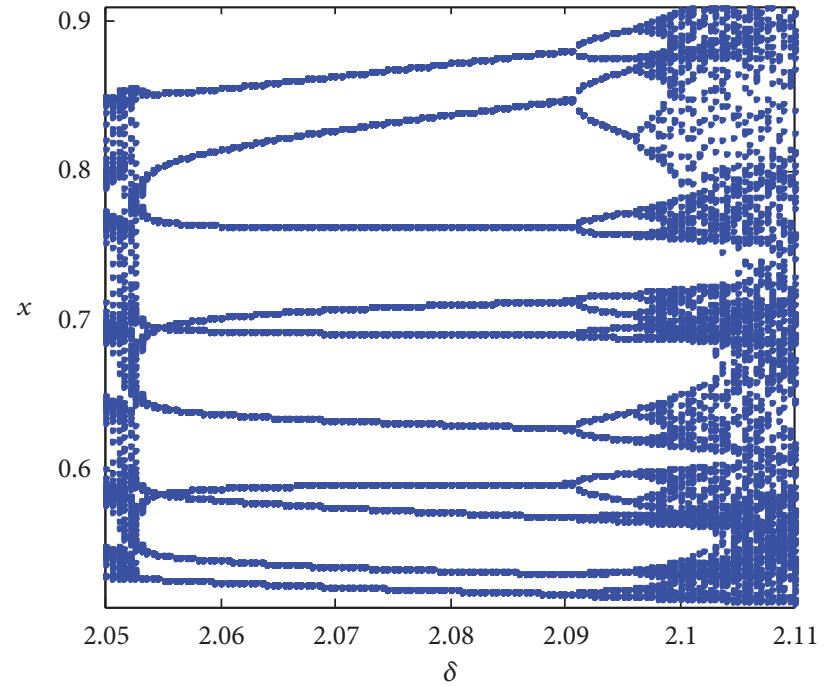

(d)

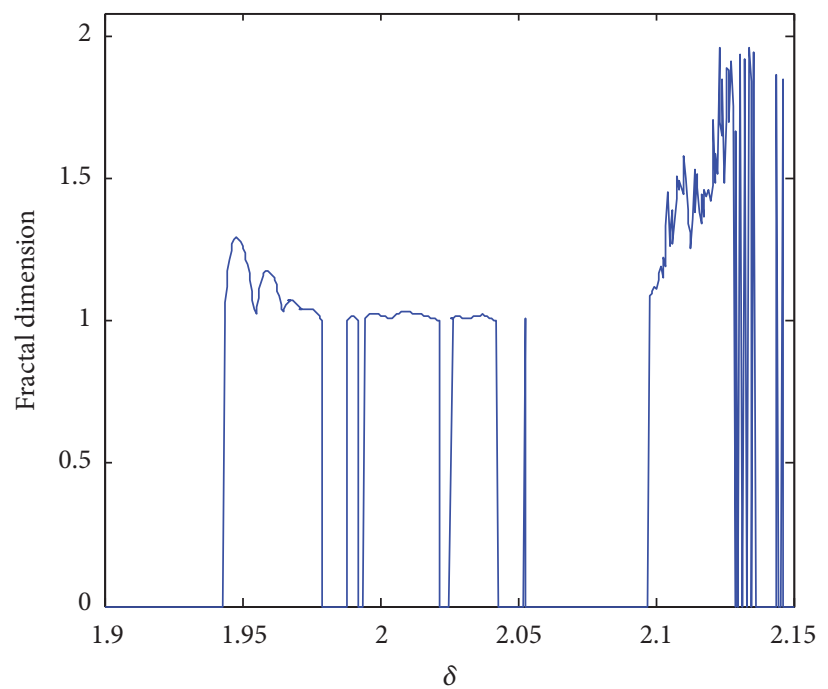

(e)

FIGURE 2: NS bifurcation and Lyapunov exponent of system (4). (a) NS bifurcation for prey, (b) NS bifurcation for predator, (c) maximum Lyapunov exponents related to (a-b), (d) local amplification diagram in (a) for $\delta \in[2.05,2.11]$, and (e) fractal dimension associated with (a). Initial value $\left(x_{0}, y_{0}\right)=(0.65,0.95)$. 

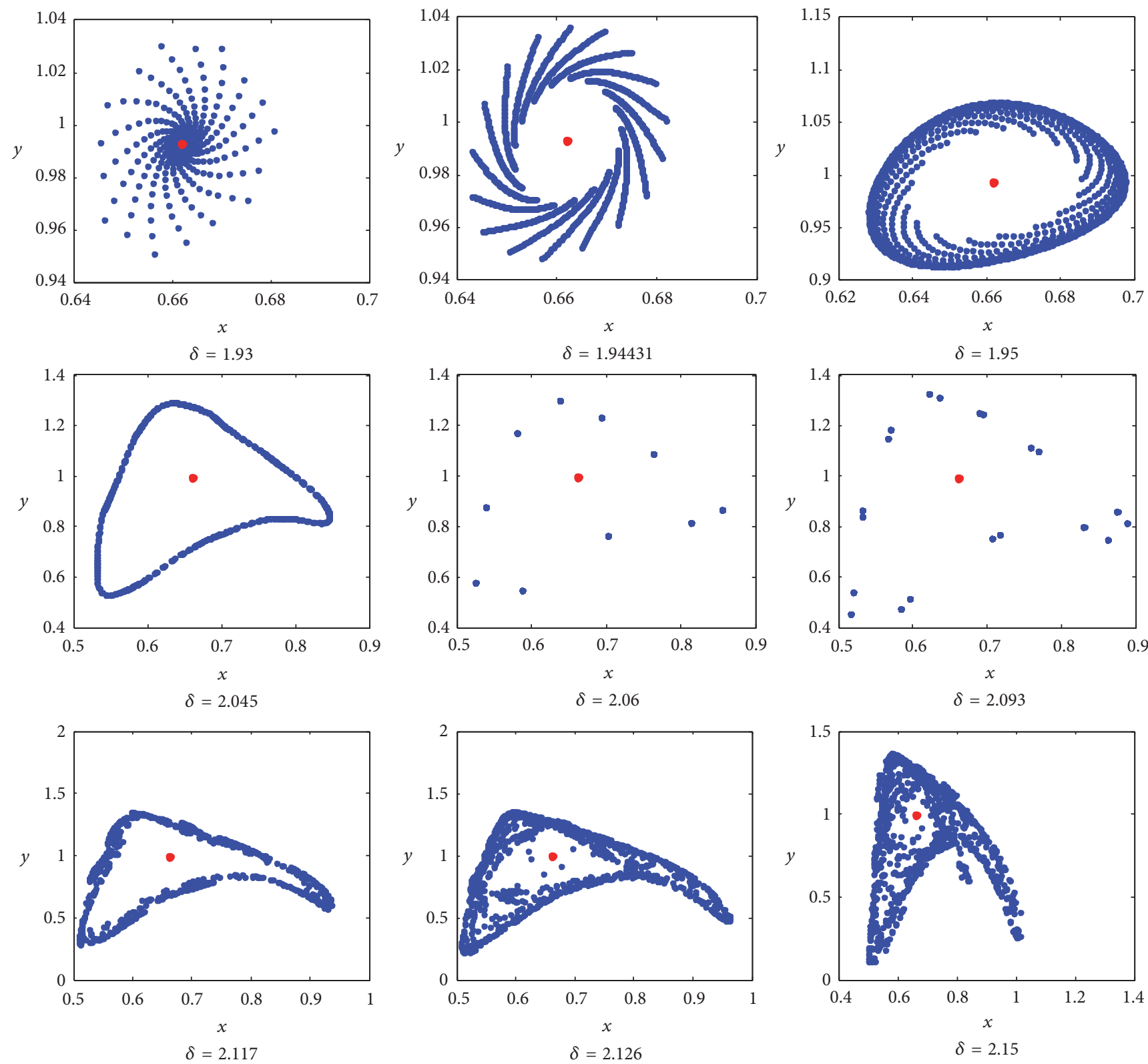

FIgURE 3: Phase portraits of bifurcation diagrams Figures 2(a) and 2(b) for different values of $\delta$.

Lyapunov exponents in Figure 2(c) relating bifurcation in Figures 2(a) and 2(b), which confirm the existences of chaos and period window as parameter $\delta$ varying. When $\delta \sim 2.126$, the sign of maximum Lyapunov exponent is confirming presence of chaos. Figure $2(\mathrm{~d})$ is local amplification of Figure 2(a) for $\delta \in[2.05,2.11]$.

The phase portraits of bifurcation diagrams in Figures 2(a) and 2(b) for different values of $\delta$ are displayed in Figure 3, which clearly illustrates the act of smooth invariant curve how it bifurcates from the stable fixed point and increases its radius. As $\delta$ grows, disappearance of closed curve occurs suddenly and a period 10 and 20 orbits appear at $\delta \sim 2.06$ and $\delta \sim 2.093$, respectively. We also see that a fully developed chaos in system $(4)$ occurs at $\delta \sim 2.15$.
For Case 3, the dynamic complexity of system (4) can be observed when more parameters vary. The diagrams of bifurcation of system (4) for control parameters $\delta \epsilon$ $[1.9,2.15]$ and $\beta \in[0.5,0.8]$ and fixing remaining parameters as in Case 2 are shown in Figures $4(\mathrm{a})$ and $4(\mathrm{~b})$. The 3D maximum Lyapunov exponent for two control parameters is plotted in Figure $4(\mathrm{c})$ and its $2 \mathrm{D}$ projection onto $(\delta, \beta)$ plane is shown in Figure 4(d). It is easy to find values of control parameters for which the dynamics of system (4) are in status of nonchaotic, periodic, or chaotic. For instance, there are chaotic dynamics for $\delta=2.15$ and $\beta=0.5$ and the nonchaotic dynamics for $\delta=1.93$ and $\beta=0.5$ (see Figure 3), which are compatible with the signs of maximum Lyapunov exponents in Figure 4(c). This exhibits that the 


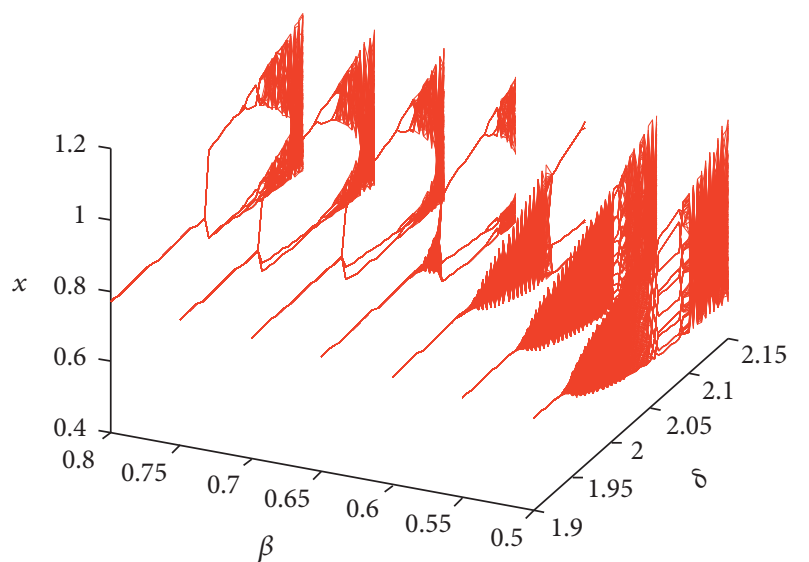

(a)

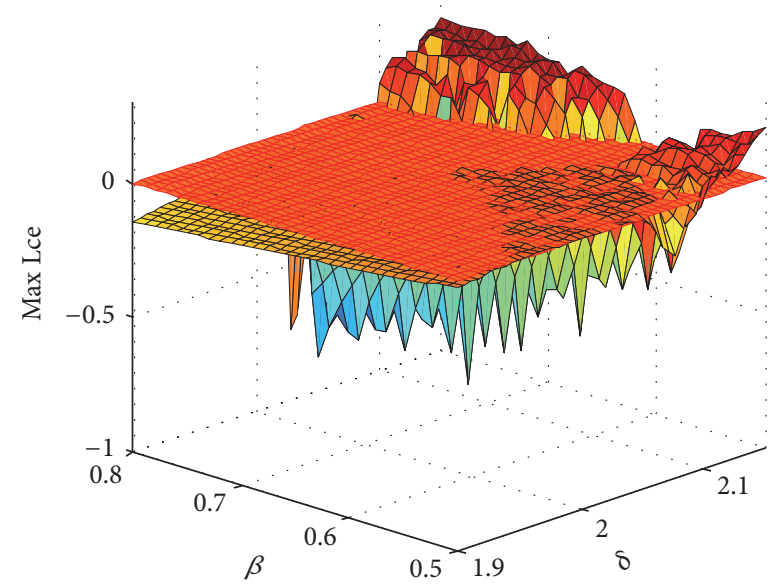

(c)

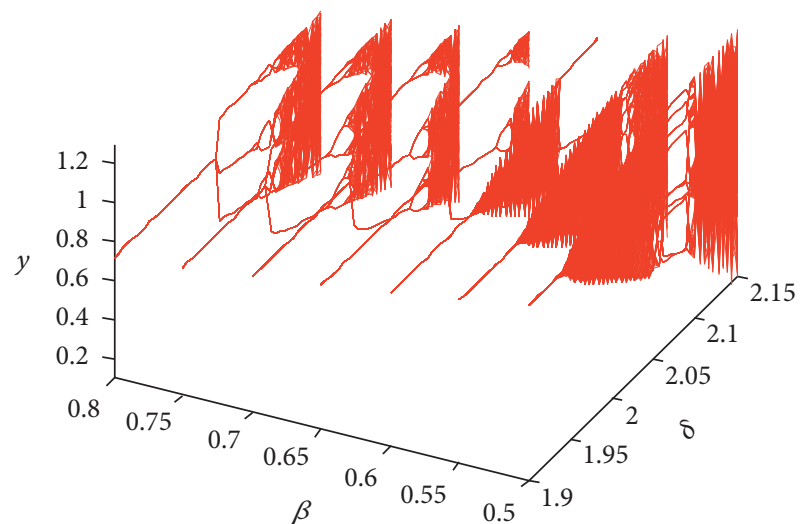

(b)

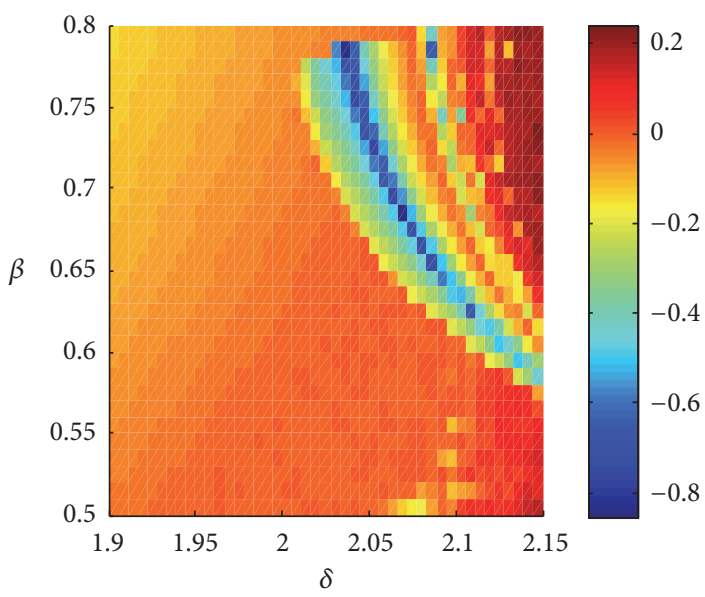

(d)

Figure 4: Diagnostic of system (4) for control parameters $\delta$ and $\beta$. (a-b) Bifurcation for prey and predator covering $\delta \in[1.9,2.15], \beta=$ $0.5,0.55,0.6,0.65,0.7,0.75,0.8 \in[0.5,0.8]$ in $(\delta-\beta-x)$ space and $(\delta-\beta-y)$ space. (c) The 3D view of maximum Lyapunov exponents related to $(\mathrm{a}-\mathrm{b})$. (d) The $2 \mathrm{D}$ projection onto $(\delta, \beta)$ plane. Initial value $\left(x_{0}, y_{0}\right)=(0.65,0.95)$.

parameter $\beta$ may play a role for chaotic dynamics in the system.

4.1. Fractal Dimension of the Map. The measure of fractal dimensions characterizes the strange attractors of a system. By using Lyapunov exponents, the fractal dimension $[18,19]$ is defined by

$$
d_{L}=j+\frac{\sum_{i=1}^{j} h_{i}}{\left|h_{j}\right|}
$$

where $h_{1}, h_{2}, \ldots, h_{n}$ are Lyapunov exponents and $j$ is the largest integer such that $\sum_{i=1}^{j} h_{i} \geq 0$ and $\sum_{i=1}^{j+1} h_{i}<0$. For our two-dimensional system (4), the fractal dimension takes the form

$$
d_{L}=1+\frac{h_{1}}{\left|h_{2}\right|}, \quad h_{1}>0>h_{2} .
$$

With parameter values as in Case 2, the fractal dimension of system (4) is plotted in Figure 2(e). The strange attractors given in Figure 3 and its corresponding fractal dimension illustrate that the Leslie type predator-prey system (4) has a chaotic dynamics as the parameter $\delta$ increases.

\section{Chaos Control}

To stabilize chaos at the state of unstable trajectories of system (4), a state feedback control method [17, 20] is applied. By adding a feedback control law as the control force $u_{n}$ to system (4), the controlled form of system (4) becomes

$$
\begin{aligned}
x_{n+1} & =x_{n}+\delta x_{n}\left[\left(1-x_{n}\right)-\frac{y_{n}}{x_{n}^{2}+a}\right]+u_{n}, \\
y_{n+1} & =y_{n}+\delta y_{n}\left[\alpha-\frac{\beta y_{n}}{x_{n}}\right], \\
u_{n} & =-k_{1}\left(x_{n}-x^{*}\right)-k_{2}\left(y_{n}-y^{*}\right),
\end{aligned}
$$

where the feedback gains are denoted by $k_{1}$ and $k_{2}$ and $\left(x^{*}, y^{*}\right)$ represent positive fixed point of system (4). 


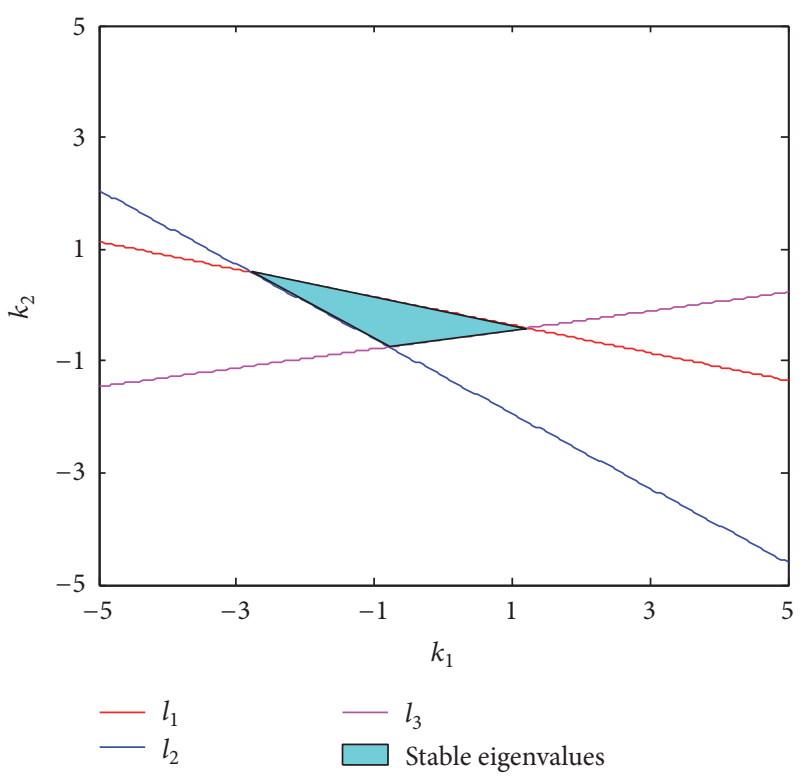

(a)
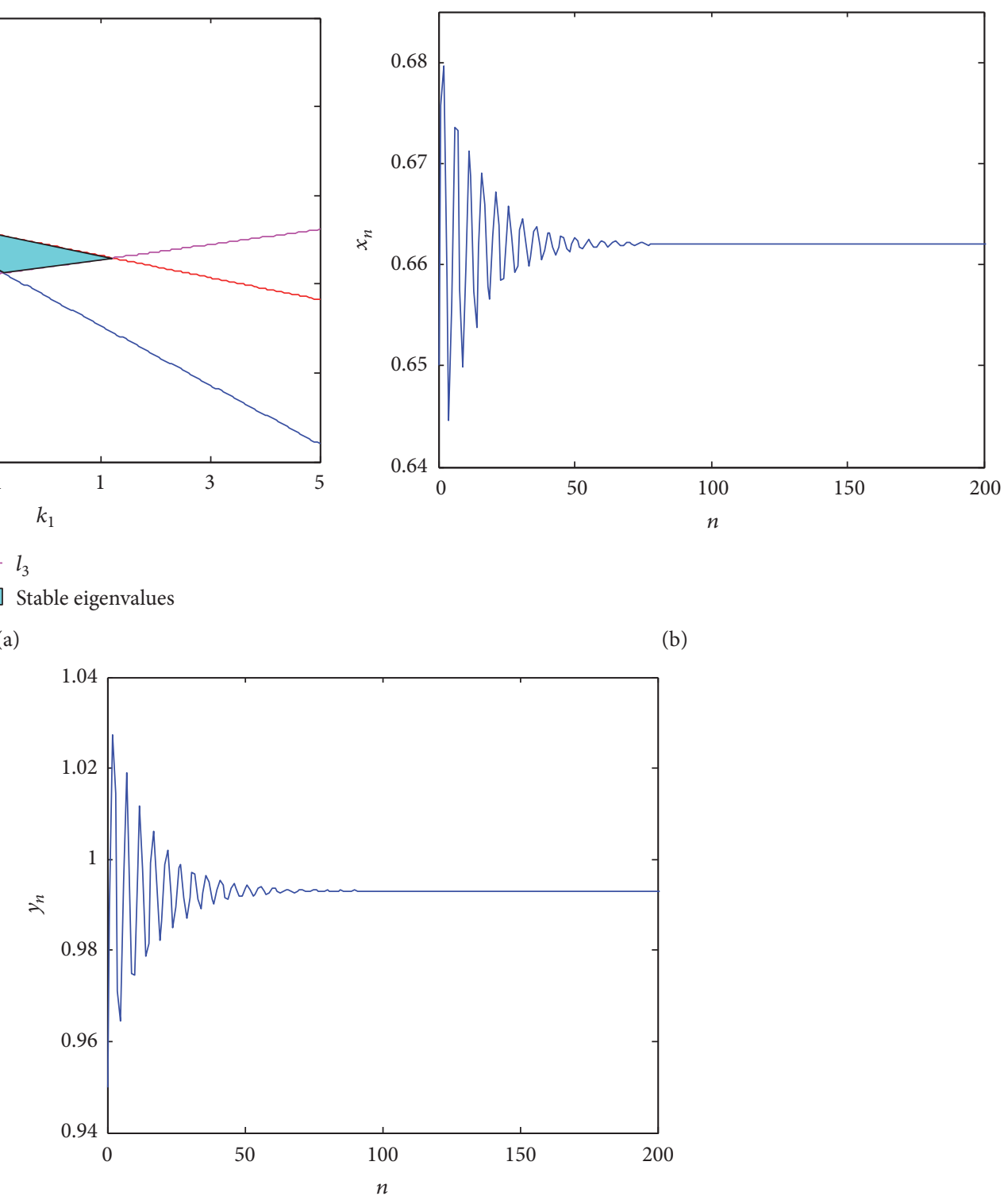

(c)

FIGURE 5: Control of chaotic trajectories of system (43). (a) Stability region in $\left(k_{1}, k_{2}\right)$ plane. (b-c) Time series for states $x$ and $y$, respectively.

The Jacobian matrix $J_{c}$ of the controlled system (43) is given by

$$
J_{c}\left(x^{*}, y^{*}\right)=\left(\begin{array}{cc}
a_{11}-k_{1} & a_{12}-k_{2} \\
a_{21} & a_{22}
\end{array}\right),
$$

where $a_{11}=1+\delta-2 \delta x+\delta y\left(x^{2}-a\right) /\left(x^{2}+a\right)^{2}, a_{12}=-\delta x /\left(x^{2}+\right.$ a), $a_{21}=\beta \delta y^{2} / x^{2}$, and $a_{22}=1+\alpha \delta-2 \beta \delta y / x$ are evaluated at $\left(x^{*}, y^{*}\right)$. The characteristic equation of $(44)$ is

$$
\lambda^{2}-\left(\operatorname{tr} J_{c}\right) \lambda+\operatorname{det} J_{c}=0
$$

where $\operatorname{tr} J_{c}=a_{11}+a_{22}-k_{1}$ and det $J_{c}=a_{22}\left(a_{11}-k_{1}\right)-a_{21}\left(a_{12}-\right.$ $\left.k_{2}\right)$. Let $\lambda_{1}$ and $\lambda_{2}$ be the roots of (45). Then

$$
\begin{aligned}
\lambda_{1}+\lambda_{2} & =a_{11}+a_{22}-k_{1}, \\
\lambda_{1} \lambda_{2} & =a_{22}\left(a_{11}-k_{1}\right)-a_{21}\left(a_{12}-k_{2}\right) .
\end{aligned}
$$

The solution of the equations $\lambda_{1}= \pm 1$ and $\lambda_{1} \lambda_{2}=1$ determines the lines of marginal stability. These conditions confirm that $\left|\lambda_{1,2}\right|<1$. Suppose that $\lambda_{1} \lambda_{2}=1$, then from (47) we have

$$
l_{1}: a_{22} k_{1}-a_{21} k_{2}=a_{11} a_{22}-a_{12} a_{21}-1 .
$$

Assume that $\lambda_{1}=1$, then from (46) and (47) we get

$$
\begin{aligned}
l_{2}: & \left(1-a_{22}\right) k_{1}+a_{21} k_{2} \\
& =a_{11}+a_{22}-1-a_{11} a_{22}+a_{12} a_{21} .
\end{aligned}
$$

Next, assume that $\lambda_{1}=-1$, then from (46) and (47) we obtain

$$
\begin{aligned}
l_{3}: & \left(1+a_{22}\right) k_{1}-a_{21} k_{2} \\
& =a_{11}+a_{22}+1+a_{11} a_{22}-a_{12} a_{21} .
\end{aligned}
$$


Then the lines $l_{1}, l_{2}$, and $l_{3}$ (see Figure 5(a)) in the $\left(k_{1}, k_{2}\right)$ plane determine a triangular region which keeps eigenvalues with magnitude less than 1.

In order to check how the implementation of feedback control method works and controls chaos at unstable state, we have performed numerical simulations. Parameter values are fixed as $\delta=2.126$ and rest as in Case 2. The initial value is $\left(x_{0}, y_{0}\right)=(0.65,0.95)$, and the feedback gains are $k_{1}=-1.3$ and $k_{2}=0.16$. Figures $5(\mathrm{~b})$ and $5(\mathrm{c})$ show that, at the fixed point $(0.662032,0.993048)$, the chaotic trajectory is stabilized.

\section{Discussions}

We investigate complex behaviors of discretized Leslie type predator-prey system (4) with simplified Holling type IV functional response in the closed first quadrant $\mathbb{R}_{+}^{2}$. By using center manifold theorem and bifurcation theory we establish that system (4) can undergo a bifurcation (flip or NS) at unique positive fixed point if $\delta$ varies around the sets $\mathrm{FB}_{E_{2}}^{1}$ or $\mathrm{FB}_{E_{2}}^{2}$ and $\mathrm{NSB}_{E_{2}}$. Numerical simulations present unpredictable behaviors of the system through a flip bifurcation which includes orbits of period 2 , period 4 , period 6 , and period 8 and through a NS bifurcation which includes an invariant cycle, orbits of period 10 and period 20, and chaotic sets, respectively. These indicate that, at the state of chaos, the system is unstable and particularly, the predator goes to extinct or goes to a stable fixed point when the dynamic of prey is chaotic. We confirm about the existence of chaos through the computation of maximum Lyapunov exponents and fractal dimension. Moreover, system (4) exhibits rich and chaotic dynamics by the variation of two control parameters and one can directly observe the chaotic phenomenon from this two-dimensional parameter-space. Finally, the chaotic trajectories at unstable state are controlled by implementing the strategy of feedback control. However, it is still a challenging problem to explore multiple parameter bifurcation in the system. We expect to obtain some more analytical results on this issue in the future.

\section{Conflicts of Interest}

The authors declare that there are no conflicts of interest.

\section{References}

[1] J. Huang, X. Xia, X. Zhang, and S. Ruan, "Bifurcation of codimension 3 in a predator-prey system of leslie type with simplified holling type IV functional response," International Journal of Bifurcation and Chaos in Applied Sciences and Engineering, vol. 26, no. 2, Article ID 1650034, 2016.

[2] Y. Li and D. Xiao, "Bifurcations of a predator-prey system of holling and leslie types," Chaos, Solitons and Fractals, vol. 34, no. 2, 2007.

[3] Z. He and X. Lai, "Bifurcation and chaotic behavior of a discretetime predator-prey system," Nonlinear Analysis. Real World Applications. An International Multidisciplinary Journal, vol. 12, no. 1, pp. 403-417, 2011.
[4] Z. He and B. Li, "Complex dynamic behavior of a discrete-time predator-prey system of holling-III type," Advances in Difference Equations, vol. 180, 2014.

[5] S. M. S. Rana, "Bifurcation and complex dynamics of a discretetime predator-prey system with simplified Monod-Haldane functional response," Advances in Difference Equations, vol. 345, 2015.

[6] S. M. S. Rana, "Bifurcation and complex dynamics of a discretetime predator-prey system," Computational Ecology and Software, vol. 5, no. 2, pp. 187-200, 2015.

[7] W. Tan, J. Gao, and W. Fan, "Bifurcation analysis and chaos control in a discrete epidemic system," Discrete Dynamics in Nature and Society. An International Multidisciplinary Research and Review Journal, Article ID 974868, 2015.

[8] Z. He and B. Li, "Complex dynamic behavior of a discrete-time predator-prey system of holling-III type," Advances in Difference Equations, vol. 191, 2014.

[9] M. Zhao, C. Li, and J. Wang, "Complex dynamic behaviors of a discrete-time predator-prey system," The Journal of Applied Analysis and Computation, vol. 7, no. 2, pp. 478-500, 2017.

[10] S. M. S. Rana, "Chaotic dynamics and control of discrete ratiodependent predator-prey system," Discrete Dynamics in Nature and Society, 2017.

[11] D. Hu and H. Cao, "Bifurcation and chaos in a discrete-time predator-prey system of holling and leslie type," Communications in Nonlinear Science and Numerical Simulation, vol. 22, pp. 702-715, 2015.

[12] H. Cao, Z. Yue, and Y. Zhou, "The stability and bifurcation analysis of a discrete holling-tanner model," Advances in Difference Equations, vol. 330, 2013.

[13] Y. A. Kuznetsov, Elements of Applied Bifurcation Theory, Springer-Verlag, New York, NY, USA, 2nd edition, 1998.

[14] J. Guckenheimer and P. Holmes, Nonlinear Oscillations, Dynamical Systems, and Bifurcation of Vector Fields, Springer-Verlag, New York, NY, USA, 1983.

[15] C. Robinson, Dynamical Systems: Stability, Symbolic Dynamics and Chaos, CRC Press, Boca Raton, Fla, USA, 2nd edition, 1999.

[16] S. Wiggins, Introduction to Applied Nonlinear Dynamical Systems and Chaos, vol. 2, Springer-Verlag, New York, NY, USA, 2003.

[17] S. N. Elaydi, An Introduction to Difference Equations, SpringerVerlag, New York, NY, USA, 1996.

[18] J. H. Cartwright, "Nonlinear stiffness, Lyapunov exponents, and attractor dimension," Physics Letters. A, vol. 264, no. 4, pp. 298302, 1999.

[19] J. L. Kaplan and J. A. Yorke, "Preturbulence: a regime observed in a fluid flow model of Lorenz," Communications in Mathematical Physics, vol. 67, no. 2, pp. 93-108, 1979.

[20] S. Lynch, Dynamical Systems with Applications Using Mathematica, Birkhäuser, Boston, Mass, USA, 2007. 


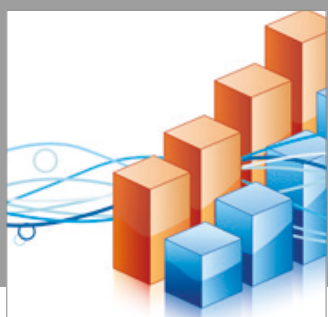

Advances in

Operations Research

vatersals

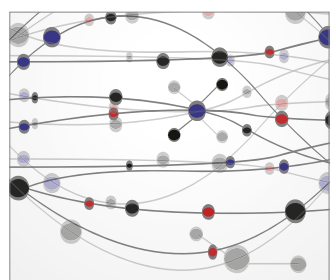

\section{The Scientific} World Journal
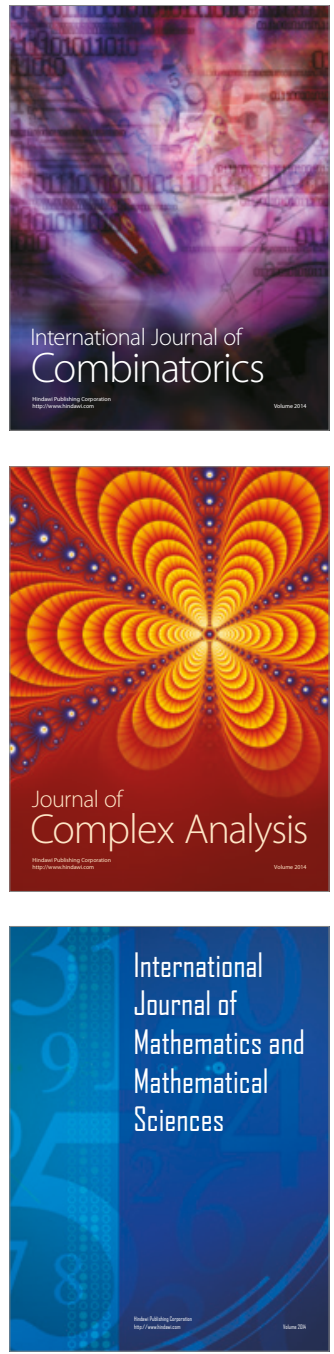
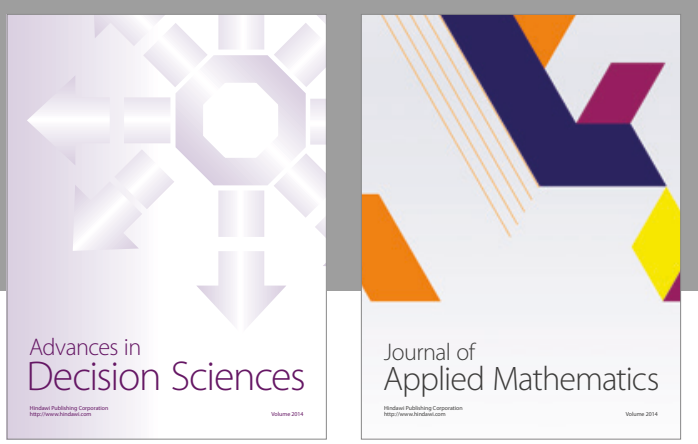

Algebra

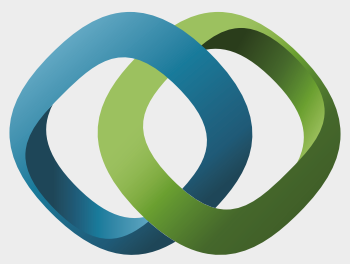

\section{Hindawi}

Submit your manuscripts at

https://www.hindawi.com
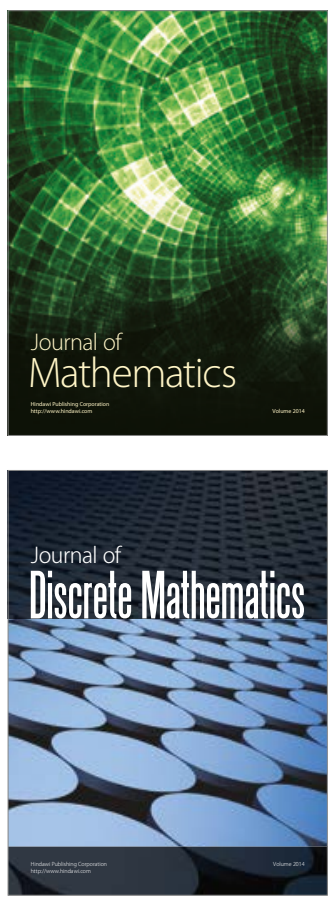

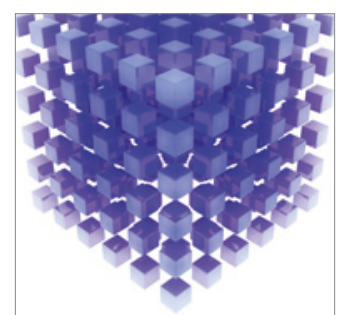

Mathematical Problems in Engineering
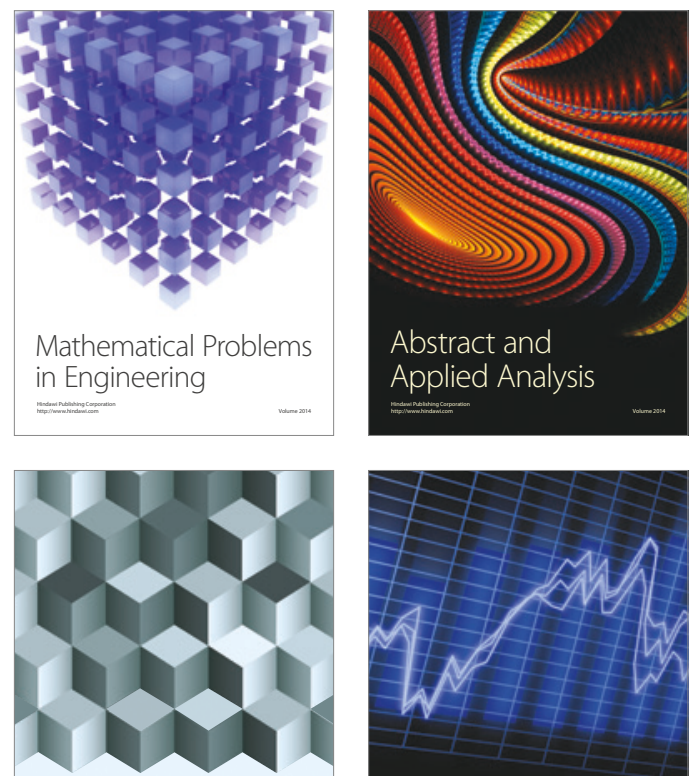

Journal of

Function Spaces

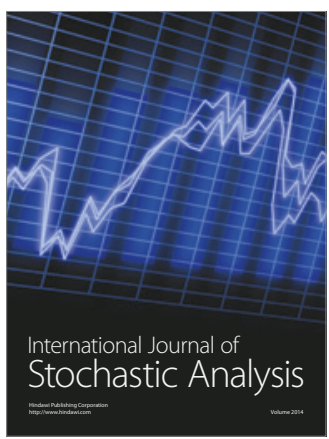

Probability and Statistics
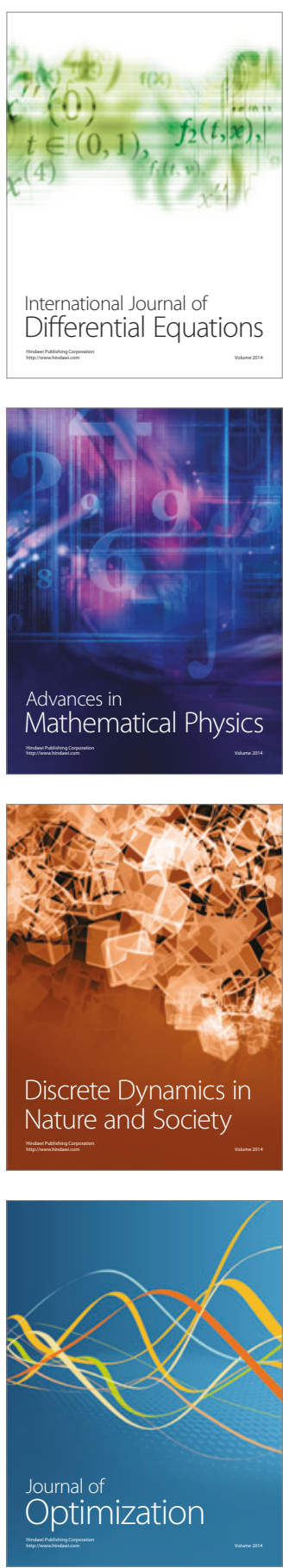